\title{
Immediate versus delayed induction of labour in hypertensive disorders of pregnancy: a systematic review and meta- analysis
}

\author{
Jia Li ${ }^{1,2,3,4}$, Xuecheng Shao', Shurong Song ${ }^{1 *}\left(\mathbb{D}\right.$, Qian Liang ${ }^{1}$, Yang Liu ${ }^{1}$ and Xiaojin Qi ${ }^{1}$
}

\begin{abstract}
Background: Mothers with hypertensive disorder of pregnancy can be managed with either immediate or delayed induction of labour with expectant monitoring of both mother and baby. There are risks and benefits associated with both the type of interventions. Hence, this review was conducted to compare outcomes of immediate and delayed induction of labour among women with hypertensive disorder of pregnancy based on disease severity and gestational age.
\end{abstract}

Methods: We conducted systematic searches in various databases including Medline, Cochrane Controlled Register of Trials (CENTRAL), Scopus, and Embase from inception until October 2019.Cochrane risk of bias tool was used to assess the quality of published trials. A meta-analysis was performed with random-effects model and reported pooled Risk ratios (RR) with 95\% confidence intervals (Cls).

Results: Fourteen randomized controlled trials with 4244 participants were included. Majority of the studies had low or unclear bias risks. Amongst late onset mild pre-eclampsia patients, the risk of renal failure was significantly lower with immediate induction of labour (pooled RR: $0.36 ; 95 \% \mathrm{Cl}: 0.14$ to 0.92 ). In severe pre-eclampsia patients, immediate induction of labour significantly reduced the risk of having small-for-gestational age babies compared to delayed induction of labour (pooled RR: 0.49; 95\%Cl: 0.29-0.84).Delayed induction was found to significantly reduce the risk of neonatal respiratory distress syndrome risk among late onset mild pre-eclampsia patients (pooled RR: 2.15; $95 \% \mathrm{Cl}: 1.14$ to 4.06 ) None of the other outcomes demonstrated statistically significant difference between the two interventions.

Conclusion: Delayed induction of labour with expectant monitoring may not be inferior to immediate induction of labour in terms of neonatal and maternal outcomes. Expectant approach of management for late onset mild preeclampsia patients may be associated with decreased risk of neonatal respiratory distress syndrome, while immediate induction of labour among severe pre-eclampsia patients is associated with reduced risk of small-forgestational age babies and among mild pre-eclampsia patients, it is associated with reduced risk of severe renal impairment.

Keywords: Delayed induction, Immediate induction, Hypertensive disorder of pregnancy, Preeclampsia

\footnotetext{
* Correspondence: Songshurong123@outlook.com

'Department of Obstetrics, The Third Central Hospital of Tianjin, 83 Jintang Road, Hedong District, Tianjin 300170, China

Full list of author information is available at the end of the article
}

C C The Author(s). 2020 Open Access This article is licensed under a Creative Commons Attribution 4.0 International License, which permits use, sharing, adaptation, distribution and reproduction in any medium or format, as long as you give appropriate credit to the original author(s) and the source, provide a link to the Creative Commons licence, and indicate if changes were made. The images or other third party material in this article are included in the article's Creative Commons licence, unless indicated otherwise in a credit line to the material. If material is not included in the article's Creative Commons licence and your intended use is not permitted by statutory regulation or exceeds the permitted use, you will need to obtain permission directly from the copyright holder. To view a copy of this licence, visit http://creativecommons.org/licenses/by/4.0/ The Creative Commons Public Domain Dedication waiver (http://creativecommons.org/publicdomain/zero/1.0/) applies to the data made available in this article, unless otherwise stated in a credit line to the data. 


\section{Background}

Hypertensive disorders during pregnancy are seen in approximately $3-10 \%$ of all pregnant women and can lead to serious maternal as well as neonatal morbidity and mortality [1-3].Globally, around 80-120 women die every day because of hypertensive complication during pregnancy [4].Mothers affected by the disorder are at higher risk of developing eclampsia with seizures, renal failure, liver failure, difficulty in breathing and HELLP syndrome (Haemolysis, elevated liver enzymes and low platelet count) [5-8].

Immediate delivery of the placenta remains the only definitive management for this condition. However, delivery of preterm baby is associated with increased perinatal mortality and other short and long-term perinatal complications like respiratory distress syndrome (RDS), neonatal seizures, intracerebral haemorrhage etc. [9-13]. Another area of concern is that women with immediate induction of labour have higher caesarean section rates [14].

Alternatively, the disorder can be managed by expectant monitoring of mother and baby and delaying the labour. Expectant management consists of frequent monitoring of blood pressure, maternal symptoms (like headache, abdominal pain, blurring of vision, decrease in foetal movements, vaginal bleeding), liver \& renal function test and complete blood count depending on the severity of the disease. Indications for delivery of these women include poor blood pressure control despite treatment, developing eclamptic maternal symptoms like headache, visual disturbances, epigastric pain, nausea and vomiting, unfavourable blood tests result and decrease in foetal movements. However, this line of management is associated with increased maternal morbidity and mortality wherein the disease may progress to preeclampsia or eclampsia, HELLP syndrome or abruptio placenta $[15,16]$.

In addition, maternal and neonatal outcomes with wither modes of intervention may vary depending on the severity of hypertension and gestational age [17-19]. American College of Obstericians and Gynecologists (ACOG) taskforce bulletin has indicated that a woman with pre-eclampsia should be delivered at 37 weeks of gestation [19]. Though, preterm delivery is considered as an option for severe pre-eclampsia, patients should be carefully evaluated for the adverse neonatal outcomes associated with the immediate induction of labour. Also, controversy exists on the benefits of having an elective delivery over delayed induction before 34 weeks of gestation. ACOG taskforce bulletin has stated that "continued pregnancy can be undertaken at facilities only if there are adequate intensive care facilities for both mother and neonate at less than 34 weeks of gestation". It does not provide further instructions on to whether an elective delivery needs to be performed if complications like oliguria or anuria, swelling of feet, pulmonary edema and cerebral or visual disturbances occurs [19]. Despite these recommendations, several clinicians consider that delayed induction of labour as pre-eclampsia intervention after the 34 weeks of gestation will promote a better outcome for both mothers and neonates. However, evidences supporting the management criteria are very limited.

As both the immediate and delayed induction have their own advantages and disadvantages, there is a need for high-level evidence to assess which intervention results in better outcomes for hypertensive disorder during pregnancy. Hence, the purpose of this meta-analysis is to compare maternal and neonatal outcomes following immediate induction of labour vs delayed induction for hypertensive disorder of pregnancy based on severity and gestational age.

\section{Methods \\ Inclusion criteria}

We included only randomized controlled trials (RCTs) for the current review. The inclusion criteria were as follows: studies on women with hypertensive disorder of pregnancy irrespective of disease severity and gestational age; studies comparing immediate induction of labour and delayed labour with expectant monitoring of mother and baby; studies outcomes including any of the following.

\section{Maternal outcomes}

Maternal mortality (death during pregnancy or up to 42 days after delivery), maternal morbidity (eclampsia, renal failure, HELLP syndrome, thromboembolic disease, postpartum haemorrhage, caesarean section rate, placental abruption).

\section{Foetal/neonatal outcomes}

Stillbirth rate (death of foetus at or after 28 weeks of gestation), perinatal mortality (death of foetus after 28 weeks of gestation till 7 days after delivery), neonatal mortality (death within 28 days of birth), neonatal morbidity (respiratory distress syndrome, neonatal seizures, small for gestational age, neonatal intensive care unit admission, necrotizing enterocolitis, intraventricular haemorrhage).

Non-randomised studies, single arm studies, abstracts, review articles were excluded.

\section{Search strategy}

Relevant articles for this study were identified by searching Medline, Embase, Cochrane Central Register of Controlled Trials (CENTRAL), Scopus, ScienceDirect and Google scholar databases and search engines for studies conducted from the inception (January 1964) to 
October 2019. Only papers published in English were included. Trial registries such as WHO International Clinical Trials Registry Platform (ICTRP) and ClinicalTrials. gov were also searched The following medical subject headings $(\mathrm{MeSH})$ were used in combination with the free text terms: "pregnancy induced hypertension" OR "hypertensive disorder of pregnancy" OR "gestational hypertension" OR "pre-eclampsia" OR "hypertension" AND ("immediate delivery" OR "immediate induction of labour" OR "induction of labour") AND ("expectant management" OR "delayed induction of labour") AND "pregnancy" OR "pregnant women", with the limits to 'human' subjects and study design 'randomized controlled trial'. .

All relevant studies were analyzed separately by two reviewers based on the inclusion criteria listed above. The analysis was done first at the title and abstract level and then at the full-text level. Any disagreement was resolved by discussion with a third reviewer. Additionally, reference list of full-text articles was searched for any missed-out studies. The Preferred Reporting Items for Systematic Review and Meta-Analysis (PRISMA) check list was used for reporting the current review [17].

\section{Data collection}

The data extracted from the included studies contained all the details necessary for quality of study assessment, including: title and authors, study design and setting, participants, sample size, inclusion and exclusion criteria, characteristics of intervention and comparison groups, duration of follow up, primary and secondary study outcomes. Corresponding authors were contacted by email for missing data or when clarification or additional information was required for the methodological assessment of the included studies.

Outcome data were independently extracted by primary and secondary authors. In case of studies reporting multiple arms in a single trial, only the relevant arms were included. Collected data were transferred into the statistical software RevMan (ver 5.3) by the first author, and double checked by the third author.

\section{Risk of bias assessment}

The risk of bias for included studies was independently assessed by two authors using Cochrane risk of bias tool for RCTs [18]. The risk of bias was assessed by: generation of random sequence, allocation concealment, blinding of the participants and outcome assessment, incomplete outcome data and selective outcome reporting.

Risk of bias was graded based on the scoring of abovementioned domains; Grading was done as low, high or unclear based on the adequacy of information and satisfaction of the criteria.

\section{Statistical analysis}

Meta-analysis was performed using RevMan 5.3 (Copenhagen: The Nordic Cochrane Centre, The Cochrane Collaboration, 2014). The pooled effect for the dichotomous outcomes was estimated by retrieving number of events and number of participants for each study group to calculate Relative Risk- RR. Meta-analysis was performed separately for studies with mild to moderate pre-eclampsia and severe pre-eclampsia as defined by the individual studies. Further subgroup analysis under each of these domains was carried out based on the gestational age of onset (i.e. early or late onset). Random-effects model with inverse variance was used. In case of missing data, the corresponding author of the included trial was contacted. Alternatively, imputation methods were used to fill in the missing data. Heterogeneity and inconsistencies among the included studies were assessed by chi square test and $\mathrm{I}^{2}$ statistics respectively. Results were graphically represented by forest plot. Selected studies were assessed for the reporting bias was assessed by comparing list of outcomes in the full study protocol with that of the published trial, where available.

\section{Results}

\section{Search results}

In total, 994 citations were identified, of which 409were retrieved from Medline, 312 from Scopus, 209 from Embase, 55 from CENTRAL, 6 from ClinicalTrials.gov and 3 from WHO ICTRP. One hundred twelve relevant studies were screened by their full texts. Bibliographies of these articles were reviewed and 3additional studies were identified. Finally, 14 studies with 4244 participants satisfying the inclusion criteria were included (Fig. 1) [15, 16, 20-33].

\section{Characteristics of included studies}

Characteristics of the included studies are described in Table 1. All the included studies were RCTs. Most the studies (6 out of 14) were conducted in Europe. In total, 2132 participants in the immediate induction arm were compared with 2112 participants in the delayed induction arm. Total sample size of the studies varied from 30 to 946 while sample size in immediate induction arm varied from 15 to 471 and in delayed induction arm varied from 15 to 475 . The definitions used for the diagnosis of gestational hypertension, pre-eclampsia and eclampsia are more or less similar across the studies included. However, there is a difference in the inclusion criteria based on the gestational age of onset and severity of the condition. Seven studies [15, 22-24, 27-29] were conducted among late onset pre-eclampsia and rest of the studies [16, 25, 26, 30-33] among early onset preeclampsia. Eight studies were conducted on mild to moderate pre-eclampsia patients $[15,16,22,23,26-$ 


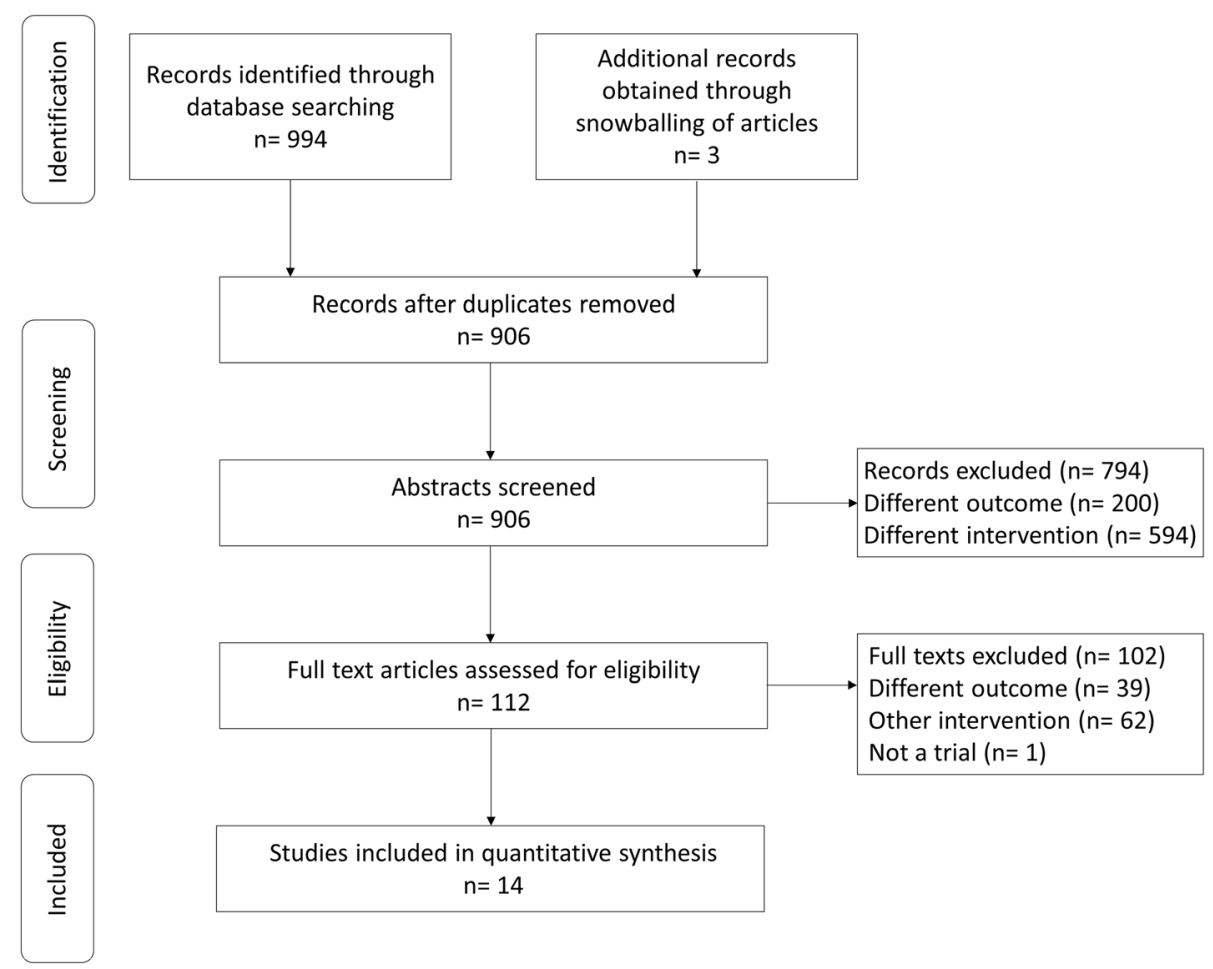

Fig. 1 PRISMA flow chart showing the selection of studies for the current review $(n=14)$

29].Six studies were conducted on severe pre-eclampsia patients [24, 25, 30-33].

\section{Quality of the included studies}

Authors assessment of risk of bias for included studies is presented in Table 2. Most of the studies had low risk of bias with respect to the randomization process (random sequence generation and allocation concealment). All the included studies had either high or unclear risk of bias with respect to blinding of participants and outcome assessment. All the studies had low or unclear risk of bias with respect to incomplete outcome data and selective reporting of outcome except Majeed et al. [29] and Mesbah EMM [30].

\section{Outcomes}

All the outcome estimates based on the gestational age of onset and severity of condition is provided in the Table 3.

\section{Maternal outcomes Maternal mortality}

Seven studies reported data on maternal mortality. Five of those studies had zero incidence of maternal mortality in both study groups Stratification based on severity and gestational age of onset had one study each under mild [15] and severe pre-eclampsia [24] and both were conducted among late onset pre-eclampsia patients.
The RR in mild pre-eclampsia group was 3.07 (95\%CI: $0.13-75.19)$ and severe pre-eclampsia group was 0.34 (0.01-8.23). Both showing non-significant difference in terms of maternal mortality (Additional file 2: Appendix $1.1 \& 1.2$ ).

\section{Maternal morbidity}

Six studies reported data on eclampsia, of which, Metaanalysis indicated no statistical significant difference in the risk of eclampsia for late onset mild pre-eclampsia [RR:0.76 (95\%CI: 0.05 to 11.18); $\mathrm{I}^{2}=34 \%$ ] (Additional file 2: Appendix 1.3) as well as for early onset severe pre-eclampsia [RR: 0.98 (95\%CI: 0.06-15.58); $\mathrm{I}^{2}=$ not applicable] (Additional file 2: Appendix 1.4). Five studies reported data on severe renal impairment. Meta-analysis indicated statistically significant reduced risk of renal failure in induction arm amongst late onset mild pre-eclampsia patients [RR: 0.36 (95\%CI: 0.14 to 0.92 ); $\mathrm{I}^{2}=$ not applicable] (Additional file 2: Appendix 1.5) but no difference for early onset severe pre-eclampsia patients [RR: 0.32 (95\%CI: 0.05-1.99); $\mathrm{I}^{2}=0 \%$ ] (Additional file 2: Appendix 1.6). Six studies reported data on incidence of HELLP syndrome among mothers following immediate or delayed induction of labour. Pooled analysis was insignificant for both late onset mild pre-eclampsia [RR:0.40 (95\%CI: 0.17 to 11.94); $\mathrm{I}^{2}=0 \%$ ] (Additional file 2: Appendix 1.7) and early onset severe pre-eclampsia 
Table 1 Characteristics of the included studies, $N=14$

\begin{tabular}{|c|c|c|c|c|c|c|c|c|c|}
\hline S.No & $\begin{array}{l}\text { Author and } \\
\text { year }\end{array}$ & Country & $\begin{array}{l}\text { Study } \\
\text { Design }\end{array}$ & Inclusion criteria & $\begin{array}{l}\text { Sample } \\
\text { size in } \\
\text { early } \\
\text { induction } \\
\text { arm }\end{array}$ & $\begin{array}{l}\text { Sample } \\
\text { size in } \\
\text { delayed } \\
\text { induction } \\
\text { arm }\end{array}$ & $\begin{array}{l}\text { Gestational } \\
\text { age of } \\
\text { onset }\end{array}$ & $\begin{array}{l}\text { Severity } \\
\text { of pre- } \\
\text { eclampsia }\end{array}$ & Intervention \\
\hline 1. & $\begin{array}{l}\text { Bhageerathy } \\
\text { et al. } 2016 \\
\text { [22] }\end{array}$ & India & $\begin{array}{l}\text { Randomized } \\
\text { Controlled } \\
\text { Trial }\end{array}$ & $\begin{array}{l}\text { All women with a } \\
\text { singleton pregnancy, } \\
\text { aged } 18 \text { to } 35 \text { years, } \\
\text { with cephalic } \\
\text { presentation at } 37 \text { to } \\
40 \text { weeks of gestation, } \\
\text { with mild gestational } \\
\text { hypertension. Systolic } \\
\text { BP between } 140 \text { and } \\
159 \text { mm of Hg and a } \\
\text { diastolic BP between } \\
90 \text { and } 100 \text { mm of Hg } \\
\text { (Korotkoff Phase V) } \\
\text { repeated after } 4 \mathrm{~h} \\
\text { were recruited }\end{array}$ & 49 & 51 & $\begin{array}{l}37-39.5 \\
\text { weeks (late } \\
\text { onset) }\end{array}$ & Mild & $\begin{array}{l}\text { Experimental } \\
\text { intervention: For } \\
\text { those in the } \\
\text { immediate induction } \\
\text { arm (group 1), a } \\
\text { vaginal examination } \\
\text { was done to assess } \\
\text { the Bishop's score. If } \\
\text { the score was } 6 \text { or } \\
\text { more, artificial rupture } \\
\text { of membranes with } \\
\text { or without oxytocin } \\
\text { augmentation was } \\
\text { done within } 12 \text { h of } \\
\text { randomization. If the } \\
\text { score was less than } 6 \text {, } \\
\text { cervical ripening was } \\
\text { done with PGE } 1 \text { ( } 25 \\
\text { microgram } 6 \text { th hourly } \\
\text { for } 2 \text { doses) as is the } \\
\text { routine for induction } \\
\text { of labour in our } \\
\text { hospital. }\end{array}$ \\
\hline
\end{tabular}

Comparison/control intervention: For those allocated to the conservative management arm (group 2), pregnancy induced hypertension $(\mathrm{PIH})$ work up which included platelet count, serum creatinine, serum transaminases (SGOT, SGPT), lactate dehydrogenase (LDH) and blood picture was done. They were advised daily home blood pressure (BP) monitoring by a local doctor or nurse who recorded it.

Pregnant women

between $36+0$ and $41+0$ weeks' gestation who had a singleton fetus in cephalic presentation, suspected intrauterine growth restriction, and who were under specialised obstetric care were recruited.

$321329 \quad \begin{aligned} & 36 \text { weeks } \\ & \text { (Late onset) }\end{aligned}$

\section{Experimental} intervention:

Participants allocated to the induction of labour group were induced within $48 \mathrm{~h}$ of randomisation. If the Bishop score at randomisation was greater than 6 , labour was induced with amniotomy and, if necessary, augmented with oxytocin

Comparison/Control intervention:

Participants allocated to the expectant monitoring group 
Table 1 Characteristics of the included studies, $N=14$ (Continued)

\begin{tabular}{|c|c|c|c|c|c|c|c|c|c|}
\hline S.No & $\begin{array}{l}\text { Author and } \\
\text { year }\end{array}$ & Country & $\begin{array}{l}\text { Study } \\
\text { Design }\end{array}$ & Inclusion criteria & $\begin{array}{l}\text { Sample } \\
\text { size in } \\
\text { early } \\
\text { induction } \\
\text { arm }\end{array}$ & $\begin{array}{l}\text { Sample } \\
\text { size in } \\
\text { delayed } \\
\text { induction } \\
\text { arm }\end{array}$ & $\begin{array}{l}\text { Gestational } \\
\text { age of } \\
\text { onset }\end{array}$ & $\begin{array}{l}\text { Severity } \\
\text { of pre- } \\
\text { eclampsia }\end{array}$ & Intervention \\
\hline
\end{tabular}

were monitored until the onset of

spontaneous labour with daily fetal movement counts and twice weekly heart rate tracings, ultrasound examination, maternal blood pressure measurement, assessment of proteinuria, laboratory tests of liver and kidney function, and full blood count.

3. Broekhuijsen Netherlands Ran et al. 2015

[23]

4. Chappell United et al. 2019 Kingdom [24]

Randomized
controlled
trial

Women were eligible

if they had

gestationa

hypertension, pre-

eclampsia, deteriorat-

ing pre-existing

hypertension, or

superimposed pre-

eclampsia, and had a gestational age of 34 weeks up to and in-

cluding 36 weeks.

Gestational

hypertension:

diastolic blood

pressure of 100

$\mathrm{mmHg}$ or more, on at

least two occasions,

$6 \mathrm{~h}$ apart, in women

without pre-existing

hypertension (defined

as a blood pressure $\geq$

$140 / 90 \mathrm{mmHg}$ before

20 weeks of

gestation).

Pre-eclampsia:

diastolic blood

pressure of $90 \mathrm{mmHg}$

or more on at least

two occasions, $6 \mathrm{~h}$

apart, combined with proteinuria, also in

women without pre-

existing hypertension.

$352 \quad 351 \quad \begin{aligned} & 34-37 \text { weeks Mild } \\ & \text { (Late onset) }\end{aligned}$

Experimental

intervention:

planned early delivery with an induction of labour started within $24 \mathrm{~h}$ after

randomisation

Control/Comparison intervention:

expectant monitoring until 37 weeks of GA

Pregnant woman was 471 eligible if she had a diagnosis of preeclampsia or superimposed pre-eclampsia (as defined by the International Society for the Study of Hypertension in Pregnancy) with a singleton or dichorionic diamniotic twin pregnancy and at least
475 Early onset Severe Experimental intervention:

induction of labour

Control/Comparison intervention:

expectant management 
Table 1 Characteristics of the included studies, $N=14$ (Continued)

\begin{tabular}{|c|c|c|c|c|c|c|c|c|c|}
\hline S.No & $\begin{array}{l}\text { Author and } \\
\text { year }\end{array}$ & Country & $\begin{array}{l}\text { Study } \\
\text { Design }\end{array}$ & Inclusion criteria & $\begin{array}{l}\text { Sample } \\
\text { size in } \\
\text { early } \\
\text { induction } \\
\text { arm }\end{array}$ & $\begin{array}{l}\text { Sample } \\
\text { size in } \\
\text { delayed } \\
\text { induction } \\
\text { arm }\end{array}$ & $\begin{array}{l}\text { Gestational } \\
\text { age of } \\
\text { onset }\end{array}$ & $\begin{array}{l}\text { Severity } \\
\text { of pre- } \\
\text { eclampsia }\end{array}$ & Intervention \\
\hline & & & & $\begin{array}{l}\text { one viable fetus, was } \\
\text { aged } 18 \text { years or } \\
\text { older, and was able } \\
\text { to give written in- } \\
\text { formed consent. } \\
\text { Women with any } \\
\text { other comorbidity (in- } \\
\text { cluding pre-existing } \\
\text { hypertension or dia- } \\
\text { betes) or with a previ- } \\
\text { ous caesarean section } \\
\text { or any foetal position } \\
\text { were eligible. }\end{array}$ & & & & & \\
\hline 5. & $\begin{array}{l}\text { Duvekot } \\
\text { et al. } 2015 \\
{[25]}\end{array}$ & Netherlands & $\begin{array}{l}\text { Randomized } \\
\text { controlled } \\
\text { Trial }\end{array}$ & $\begin{array}{l}\text { Women between } \\
28+0 \text { and } 34+0 \\
\text { weeks of gestation } \\
\text { after admission for } \\
\text { severe preeclampsia } \\
\text { with or without } \\
\text { HELLP syndrome }\end{array}$ & 25 & 30 & $\begin{array}{l}\text { 28-34 weeks } \\
\text { (Early onset) }\end{array}$ & Severe & $\begin{array}{l}\text { Experimental } \\
\text { intervention: } \\
\text { induction of labour } \\
\text { Control/Comparison } \\
\text { intervention: } \\
\text { expectant } \\
\text { management }\end{array}$ \\
\hline 6. & $\begin{array}{l}\text { GRIT study } \\
\text { group } 2013 \\
\text { [26] }\end{array}$ & $\begin{array}{l}13 \\
\text { European } \\
\text { countries }\end{array}$ & $\begin{array}{l}\text { Randomized } \\
\text { controlled } \\
\text { trial }\end{array}$ & $\begin{array}{l}\text { Singleton or multiple } \\
\text { pregnancies where } \\
\text { the responsible } \\
\text { clinician was } \\
\text { uncertain whether to } \\
\text { deliver the baby } \\
\text { immediately, the } \\
\text { gestational age was } \\
\text { between } 24 \text { and } 36 \\
\text { weeks and the } \\
\text { umbilical artery } \\
\text { Doppler waveform } \\
\text { had been recorded }\end{array}$ & 273 & 274 & $\begin{array}{l}\text { 24-36 weeks } \\
\text { (Early onset) }\end{array}$ & Mild & $\begin{array}{l}\text { Experimental } \\
\text { intervention: } \\
\text { induction of labour } \\
\text { Control/Comparison } \\
\text { intervention: } \\
\text { expectant } \\
\text { management }\end{array}$ \\
\hline 7. & $\begin{array}{l}\text { Hamed et al. } \\
2014 \text { [16] }\end{array}$ & $\begin{array}{l}\text { Saudi } \\
\text { Arabia and } \\
\text { Egypt }\end{array}$ & $\begin{array}{l}\text { Randomized } \\
\text { controlled } \\
\text { Trial }\end{array}$ & $\begin{array}{l}\text { Mild to moderate } \\
\text { essential chronic } \\
\text { hypertension without } \\
\text { proteinuria, singleton } \\
\text { pregnancy, and } \\
\text { gestational age at } \\
\text { recruitment of } 24-36 \\
\text { weeks. } \\
\text { Mild to moderate } \\
\text { chronic hypertension } \\
\text { was diagnosed if } \\
\text { diastolic blood } \\
\text { pressure was } \\
\text { between } 90 \text { and } 110 \\
\text { mmHg and/or systolic } \\
\text { pressure was } \\
\text { between } 140 \text { and } \\
160 \text { mmHg on two } \\
\text { occasions at least } 6 \mathrm{~h} \\
\text { apart in the first half } \\
\text { of pregnancy, or if } \\
\text { the patient was } \\
\text { known to be } \\
\text { hypertensive before } \\
\text { pregnancy }\end{array}$ & 38 & 38 & $\begin{array}{l}\text { 24-36 weeks } \\
\text { (early onset) }\end{array}$ & Mild & $\begin{array}{l}\text { Experimental } \\
\text { intervention: delivery } \\
\text { at } 37 \text { completed } \\
\text { weeks, provided that } \\
\text { no maternal or fetal } \\
\text { complications } \\
\text { demanded elective } \\
\text { preterm labour } \\
\text { Control/Comparison } \\
\text { intervention: } \\
\text { expectant } \\
\text { management until } \\
\text { the spontaneous } \\
\text { onset of labour or } 41 \\
\text { gestational weeks }\end{array}$ \\
\hline 8. & $\begin{array}{l}\text { Koopmans } \\
\text { et al. } 2009 \\
{[27]}\end{array}$ & Netherlands & $\begin{array}{l}\text { Randomized } \\
\text { controlled } \\
\text { Trial }\end{array}$ & $\begin{array}{l}\text { Women with a } \\
\text { singleton pregnancy } \\
\text { and a fetus in } \\
\text { cephalic presentation }\end{array}$ & 377 & 379 & $\begin{array}{l}36 \text { weeks } \\
\text { (late onset) }\end{array}$ & $\begin{array}{l}\text { Mild to } \\
\text { moderate }\end{array}$ & $\begin{array}{l}\text { Experimental } \\
\text { intervention: } \\
\text { induction of labour } \\
\text { within } 24 \mathrm{~h} \text { of }\end{array}$ \\
\hline
\end{tabular}


Table 1 Characteristics of the included studies, $N=14$ (Continued)

\begin{tabular}{|c|c|c|c|c|c|c|c|c|c|}
\hline S.No & $\begin{array}{l}\text { Author and } \\
\text { year }\end{array}$ & Country & $\begin{array}{l}\text { Study } \\
\text { Design }\end{array}$ & Inclusion criteria & $\begin{array}{l}\text { Sample } \\
\text { size in } \\
\text { early } \\
\text { induction } \\
\text { arm }\end{array}$ & $\begin{array}{l}\text { Sample } \\
\text { size in } \\
\text { delayed } \\
\text { induction } \\
\text { arm }\end{array}$ & $\begin{array}{l}\text { Gestational } \\
\text { age of } \\
\text { onset }\end{array}$ & $\begin{array}{l}\text { Severity } \\
\text { of pre- } \\
\text { eclampsia }\end{array}$ & Intervention \\
\hline & & & & $\begin{array}{l}\text { at a gestational age } \\
\text { of between } 36 \text { (0 } \\
\text { days) and } 41 \text { weeks } \\
\text { (0 days), and who had } \\
\text { gestational } \\
\text { hypertension or mild } \\
\text { pre-eclampsia. } \\
\text { Pre-eclampsia: } \\
\text { diastolic BP > } 90 \text { mm } \\
\text { on two occasions at } \\
\text { least } 6 \mathrm{~h} \text { apart; } \\
\text { proteinuria (two or } \\
\text { more occurrences of } \\
\text { protein on a dipstick, } \\
>300 \text { mg total } \\
\text { protein within a } 24-\mathrm{h} \\
\text { urine collection, or ra- } \\
\text { tio of protein to cre- } \\
\text { atinine }>30 \text { mg/ } \\
\text { mmol) } \\
\text { Gestational } \\
\text { hypertension: } \\
\text { diastolic BP } \geqq 95 \\
\text { mmHg, on two } \\
\text { occasions at least } 6 \mathrm{~h} \\
\text { apart }\end{array}$ & & & & & $\begin{array}{l}\text { randomisation } \\
\text { Control/Comparison } \\
\text { intervention: } \\
\text { expectant monitoring. } \\
\text { They were monitored } \\
\text { until the onset of } \\
\text { spontaneous delivery, } \\
\text { in hospital or } \\
\text { outpatient setting, } \\
\text { depending on the } \\
\text { condition of the } \\
\text { woman with frequent } \\
\text { blood pressure } \\
\text { measurements and } \\
\text { testing of urine for } \\
\text { protein of the } \\
\text { mother. }\end{array}$ \\
\hline 9. & $\begin{array}{l}\text { Owens et al. } \\
2014 \text { [28] }\end{array}$ & $\begin{array}{l}\text { United } \\
\text { States of } \\
\text { America }\end{array}$ & $\begin{array}{l}\text { Randomized } \\
\text { controlled } \\
\text { Trial }\end{array}$ & $\begin{array}{l}\text { Late preterm patients } \\
\text { with preeclampsia } \\
\text { without severe } \\
\text { features assigned to } \\
\text { immediate delivery/ } \\
\text { expectant } \\
\text { management until } 37 \\
\text { weeks gestation or } \\
\text { earlier if severe } \\
\text { features develop }\end{array}$ & 94 & 75 & $\begin{array}{l}\text { 34-37 weeks } \\
\text { (late onset) }\end{array}$ & Mild & $\begin{array}{l}\text { Experimental } \\
\text { intervention: } \\
\text { planned early delivery } \\
\text { via induction of } \\
\text { labour or caesarean } \\
\text { delivery within } 12 \mathrm{~h} \\
\text { of randomisation. All } \\
\text { study participants } \\
\text { were treated with } \\
\text { magnesium sulphate } \\
\text { prophylaxis } \\
\text { intrapartum and } \\
\text { immediately } \\
\text { postpartum. } 97 \\
\text { women were } \\
\text { randomised, } 3 \text { were } \\
\text { subsequently } \\
\text { excluded for not } \\
\text { meeting the inclusion } \\
\text { criteria } \\
\text { Control/Comparison } \\
\text { intervention: } \\
\text { inpatient expectant } \\
\text { management, to } 37 \\
\text { weeks' gestation } \\
\text { unless there was } \\
\text { spontaneous onset of } \\
\text { labour or rupture of } \\
\text { membranes, } \\
\text { suspected placental } \\
\text { abruption, } \\
\text { development of } \\
\text { severe PE of fetal } \\
\text { compromise. }\end{array}$ \\
\hline 10. & $\begin{array}{l}\text { Majeed et al. } \\
2014[29]\end{array}$ & India & $\begin{array}{l}\text { Randomized } \\
\text { controlled } \\
\text { Trial }\end{array}$ & $\begin{array}{l}\text { Pregnant women at } \\
36-40 \text { weeks' } \\
\text { gestation, with mild }\end{array}$ & 50 & 50 & $\begin{array}{l}\text { 36-40 weeks } \\
\text { (late onset) }\end{array}$ & Mild & $\begin{array}{l}\text { Experimental } \\
\text { intervention: } \\
\text { induction of labour }\end{array}$ \\
\hline
\end{tabular}


Table 1 Characteristics of the included studies, $N=14$ (Continued)

\begin{tabular}{|c|c|c|c|c|c|c|c|c|c|}
\hline S.No & $\begin{array}{l}\text { Author and } \\
\text { year }\end{array}$ & Country & $\begin{array}{l}\text { Study } \\
\text { Design }\end{array}$ & Inclusion criteria & $\begin{array}{l}\text { Sample } \\
\text { size in } \\
\text { early } \\
\text { induction } \\
\text { arm }\end{array}$ & $\begin{array}{l}\text { Sample } \\
\text { size in } \\
\text { delayed } \\
\text { induction } \\
\text { arm }\end{array}$ & $\begin{array}{l}\text { Gestational } \\
\text { age of } \\
\text { onset }\end{array}$ & $\begin{array}{l}\text { Severity } \\
\text { of pre- } \\
\text { eclampsia }\end{array}$ & Intervention \\
\hline & & & & $\begin{array}{l}\text { pre-eclampsia/ gesta- } \\
\text { tional hypertension } \\
\text { without proteinuria } \\
\text { Gestational } \\
\text { Hypertension: } \\
\text { systolic blood } \\
\text { pressure } \geq 140 \text { or } \\
\text { diastolic blood } \\
\text { pressure } \geq 90 \mathrm{mmHg} \\
\text { for the first time } \\
\text { during pregnancy } \\
\text { without proteinuria. } \\
\text { Mild pre-eclampsia: } \\
\text { systolic blood } \\
\text { pressure was } 140- \\
159 \mathrm{mmHg} \text { and } \\
\text { diastolic blood } \\
\text { pressure is } 90-109 \\
\mathrm{mmH} H \mathrm{accompanied} \\
\text { by proteinuria of }> \\
0.3 \mathrm{~g} \text { to }<5 \mathrm{~g} / 24 \mathrm{~h}\end{array}$ & & & & & $\begin{array}{l}\text { Control/Comparison } \\
\text { intervention: } \\
\text { expectant } \\
\text { management }\end{array}$ \\
\hline 11. & $\begin{array}{l}\text { Mesbah } \\
\text { EMM } 2003 \\
{[30]}\end{array}$ & Egypt & $\begin{array}{l}\text { Randomized } \\
\text { controlled } \\
\text { trial }\end{array}$ & $\begin{array}{l}\text { Pregnant women } \\
\text { with severe PE } \\
\text { between } 28 \text { and } 33+ \\
6 \text { days gestation. } \\
\text { Severe PE was } \\
\text { defined as a } \mathrm{BP}> \\
180 / 120 \mathrm{mmHg} \text { on } 2 \\
\text { occasions, } 30 \text { min } \\
\text { apart; or a BP } \\
\text { between } 160 \text { to 180/ } \\
110 \text { to } 120 \mathrm{mmHg} \text { on } \\
2 \text { occasions, } 6 \mathrm{~h} \text { apart. } \\
\text { All participants had }> \\
500 \text { mg of proteinuria } \\
\text { on a } 24 \mathrm{~h} \text { urine } \\
\text { collection measure }\end{array}$ & 15 & 15 & $\begin{array}{l}32 \text { weeks } \\
\text { (Early onset) }\end{array}$ & Severe & $\begin{array}{l}\text { Experimental } \\
\text { Intervention: } \\
\text { Administered } \\
\text { dexamethasone } \\
\text { phosphate; } 48 \mathrm{~h} \text { to } \\
\text { lapse before either an } \\
\text { induction of labour } \\
\text { was attempted ( } 50 \mu \text {, } \\
\text { vaginal misoprostol) } \\
\text { or caesarean section } \\
\text { after } 24 \mathrm{~h} \\
\text { Control/Comparison } \\
\text { Intervention: } \\
\text { Administered } \\
\text { dexamethasone } \\
\text { phosphate then } \\
\text { managed } \\
\text { conservatively with } \\
\text { bed rest, observations } \\
\text { and nifedipine to } \\
\text { control BP. Indications } \\
\text { for delivery were } \\
\text { imminent eclampsia, } \\
\text { deteriorating renal } \\
\text { function, spontaneous } \\
\text { preterm labour, } \\
\text { absent EDF or a non- } \\
\text { reassuring CTG reach- } \\
\text { ing } 34 \text { weeks }\end{array}$ \\
\hline 12. & $\begin{array}{l}\text { Odendaal HJ } \\
\text { et al. } 1990 \\
\text { [32] }\end{array}$ & Africa & $\begin{array}{l}\text { Randomized } \\
\text { controlled } \\
\text { trial }\end{array}$ & $\begin{array}{l}\text { Women with severe } \\
\text { PE at } 28 \text { to } 34 \text { weeks' } \\
\text { gestation. Severe PE } \\
\text { defined in } 4 \text { ways, } \\
\text { depending on BP, } \\
\text { proteinuria, and } \\
\text { symptoms. Women } \\
\text { were either already } \\
\text { admitted for bedrest } \\
\text { and later met criteria, } \\
\text { or admitted because } \\
\text { of severe PE, and } \\
\text { after } 48 \mathrm{~h} \text { stabilisation }\end{array}$ & 20 & 18 & $\begin{array}{l}\text { 28-34 weeks } \\
\text { (Early onset) }\end{array}$ & Severe & $\begin{array}{l}\text { Experimental } \\
\text { Intervention: } \\
\text { Delivery by induction } \\
\text { or caesarean section } \\
\text { depending on } \\
\text { obstetric } \\
\text { circumstances } 48 \mathrm{~h} \\
\text { after betamethasone. } \\
\text { If cervix not } \\
\text { favourable, } \\
\text { prostaglandin } \mathrm{E} 2 \\
\text { tablets. If still not } \\
\text { favourable after } 24 \mathrm{~h} \text {, }\end{array}$ \\
\hline
\end{tabular}


Table 1 Characteristics of the included studies, $N=14$ (Continued)

\begin{tabular}{|c|c|c|c|c|c|c|c|c|c|}
\hline S.No & $\begin{array}{l}\text { Author and } \\
\text { year }\end{array}$ & Country & $\begin{array}{l}\text { Study } \\
\text { Design }\end{array}$ & Inclusion criteria & $\begin{array}{l}\text { Sample } \\
\text { size in } \\
\text { early } \\
\text { induction } \\
\text { arm }\end{array}$ & $\begin{array}{l}\text { Sample } \\
\text { size in } \\
\text { delayed } \\
\text { induction } \\
\text { arm }\end{array}$ & $\begin{array}{l}\text { Gestational } \\
\text { age of } \\
\text { onset }\end{array}$ & $\begin{array}{l}\text { Severity } \\
\text { of pre- } \\
\text { eclampsia }\end{array}$ & Intervention \\
\hline & & & & met entry criteria & & & & & $\begin{array}{l}\text { caesarean section } \\
\text { Control/Comparison } \\
\text { intervention: Bed } \\
\text { rest on high-risk ob- } \\
\text { stetric ward; maternal } \\
\text { and fetal condition } \\
\text { monitored intensively; } \\
\text { BP controlled with } \\
\text { prazosin; delivery at } \\
34 \text { weeks unless indi- } \\
\text { cated earlier }\end{array}$ \\
\hline 13. & $\begin{array}{l}\text { Sibai BM } \\
\text { et al. } 1994 \\
\text { [33] }\end{array}$ & $\begin{array}{l}\text { United } \\
\text { States of } \\
\text { America }\end{array}$ & $\begin{array}{l}\text { Randomized } \\
\text { controlled } \\
\text { trial }\end{array}$ & $\begin{array}{l}\text { Women with severe } \\
\text { PE at } 28 \text { to } 32 \text { weeks' } \\
\text { gestation. Severe PE } \\
\text { defined as a } \\
\text { persistent elevation of } \\
\mathrm{BP} \geq 160 / 110 \mathrm{mmHg} \text {, } \\
\text { proteinuria }>500 \mathrm{mg} \\
\text { in } 24 \mathrm{~h} \text {, and uric acid } \\
>5 \mathrm{mg} / \mathrm{dL} \text {. }\end{array}$ & 46 & 49 & $\begin{array}{l}32 \text { weeks } \\
\text { (Early onset) }\end{array}$ & Severe & $\begin{array}{l}\text { Experimental } \\
\text { Intervention: } \\
\text { Delivery by caesarean } \\
\text { section or by } \\
\text { induction of labour, } \\
\text { on the basis of } \\
\text { obstetric condition, } \\
48 \mathrm{~h} \text { after first dose of } \\
\text { betamethasone } \\
\text { Control/Comparison } \\
\text { intervention: } \\
\text { Maternal and fetal } \\
\text { monitoring on an } \\
\text { antenatal ward. If } \\
\text { either condition } \\
\text { deteriorated, or } \\
\text { reached } 34 \text { weeks' } \\
\text { gestation, delivery } \\
\text { using the 'most } \\
\text { appropriate method' }\end{array}$ \\
\hline 15. & $\begin{array}{l}\text { Vigil De } \\
\text { Gracia et al. } \\
2013 \text { [31] }\end{array}$ & $\begin{array}{l}\text { Latin } \\
\text { America }\end{array}$ & $\begin{array}{l}\text { Randomized } \\
\text { controlled } \\
\text { trial }\end{array}$ & $\begin{array}{l}\text { Pregnant women } \\
\text { between } 28 \text { and } 33 \\
\text { weeks' gestation with } \\
\text { severe PE, severe } \\
\text { gestational } \\
\text { hypertension, and } \\
\text { super-imposed PE }\end{array}$ & 133 & 131 & $\begin{array}{l}32 \text { weeks } \\
\text { (Early onset) }\end{array}$ & Severe & $\begin{array}{l}\text { Experimental } \\
\text { intervention: Prompt } \\
\text { delivery': } \\
\text { glucocorticoid } \\
\text { therapy followed by } \\
\text { delivery in } 24-72 \mathrm{~h} \text {, } \\
\text { magnesium sulphate } \\
\text { continued until } 24 \mathrm{~h} \\
\text { after delivery } \\
\text { Control/Comparison } \\
\text { intervention: Treated } \\
\text { expectantly: } \\
\text { glucocorticoid } \\
\text { therapy followed by } \\
\text { delivery only for } \\
\text { specific maternal/ } \\
\text { fetal indications or } \\
\text { reaching } 34 \text { weeks of } \\
\text { gestation }\end{array}$ \\
\hline
\end{tabular}

[RR:1.15 (95\%CI: 0.65-2.02); $\mathrm{I}^{2}=0 \%$ ] (Additional file 2: Appendix 1.8).

Three studies reported data on the risk of thromboembolic disease. All the three studies were conducted among late onset mild pre-eclampsia patients. Metaanalysis indicated no statistically significant difference in risk of thromboembolic disease between the two groups [RR:1.60 (95\%CI: 0.20 to 12.99$) ; \mathrm{I}^{2}=0 \%$ ] (Additional file 2: Appendix 1.9).
Three studies reported data on the risk of postpartum haemorrhage. All the three studies were conducted among late onset mild pre-eclampsia patients. Metaanalysis indicated no statistically significant difference in risk of postpartum haemorrhage between the two groups [RR:0.82 (95\%CI: 0.56 to 1.19$) ; \mathrm{I}^{2}=0 \%$ ] (Additional file 2: Appendix 1.10). All the 14 studies have reported on the incidence of caesarean section among mothers in both the arms. The pooled RR for late onset mild pre- 
Table 2 Risk of bias assessment for the included studies, $N=14$

\begin{tabular}{|c|c|c|c|c|c|c|c|}
\hline S.No & Author and year & $\begin{array}{l}\text { Random sequence } \\
\text { generation }\end{array}$ & $\begin{array}{l}\text { Allocation } \\
\text { concealment }\end{array}$ & $\begin{array}{l}\text { Blinding of the } \\
\text { participants }\end{array}$ & $\begin{array}{l}\text { Blinding of } \\
\text { outcome } \\
\text { assessment }\end{array}$ & $\begin{array}{l}\text { Incomplete } \\
\text { outcome data }\end{array}$ & $\begin{array}{l}\text { Selective reporting } \\
\text { of outcome }\end{array}$ \\
\hline 1. & Bhageerathy et al. 2016 [22] & Low risk & Low risk & High risk & High risk & Low risk & Unclear risk \\
\hline 2. & Boers et al. 2010 [15] & Low risk & Low risk & High risk & Unclear risk & Low risk & Low risk \\
\hline 3. & Broekhuijsen et al. 2015 [23] & Low risk & Low risk & High risk & Unclear risk & Low risk & Low risk \\
\hline 4. & Chappell et al. 2019 [24] & Unclear risk & Unclear risk & High risk & High risk & Unclear risk & Unclear risk \\
\hline 5. & Duvekot et al. 2015 [25] & Low risk & Low risk & High risk & High risk & Low risk & Unclear risk \\
\hline 6. & GRIT study group 2013 [26] & Low risk & Unclear risk & High risk & Unclear risk & Unclear risk & Low risk \\
\hline 7. & Hamed et al. 2014 [16] & Low risk & Unclear risk & High risk & High risk & Low risk & Unclear risk \\
\hline 8. & Koopmans et al. 2009 [27] & Low risk & Low risk & High risk & Unclear risk & Low risk & Low risk \\
\hline 9. & Owens et al. 2014 [28] & Low risk & Unclear risk & High risk & Unclear risk & Unclear risk & Low risk \\
\hline 10. & Majeed et al. 2014 [29] & Unclear risk & Unclear risk & High risk & High risk & Unclear risk & High risk \\
\hline 11. & Mesbah EMM 2003 [30] & Low risk & Low risk & Unclear risk & Unclear risk & High risk & Low risk \\
\hline 12. & Odendaal HJ et al. 1990 [32] & Unclear risk & Unclear risk & Unclear risk & Unclear risk & Unclear risk & Unclear risk \\
\hline 13. & Sibai BM et al. 1994 [33] & Low risk & Low risk & Unclear risk & Unclear risk & Low risk & Low risk \\
\hline 14. & Vigil De Gracia et al. 2013 [31] & Low risk & Low risk & Unclear risk & Unclear risk & Low risk & Low risk \\
\hline
\end{tabular}

eclampsia was 0.95 (95\%CI: 0.82 to $1.09, \mathrm{I}^{2}=0 \%$ ) (Additional file 2: Appendix 1.11) and early onset mild pre-eclampsia was 2.23 (95\%CI: $0.42-11.87, \mathrm{I}^{2}=29 \%$ ). (Additional file 2: Appendix 1.12). For early onset severe pre-eclampsia, pooled RR was 0.95 (95\%CI: 0.89-1.01; $\mathrm{I}^{2}=10 \%$ ) (Additional file 2: Appendix 1.13). There was a significant publication bias as depicted by asymmetrical funnel plot (Additional file 1: Supplementary Fig. 1). Four studies have reported on placental abruption and all of them were conducted among early-onset severe pre-eclampsia patients. The pooled RR was 0.47 (95\%CI: $0.20-1.12 ; \mathrm{I}^{2}=0 \%$ ) (Additional file 2: Appendix 1.14).

\section{Foetal/ neonatal outcome}

Eight studies have reported on the stillbirth rate in both the arms. First, among mild pre-eclampsia studies, stillbirth occurred in only one study. The RR was 0.17 (95\%CI: 0.02 to $1.45 ; \mathrm{I}^{2}=$ not applicable) (Additional file 2: Appendix 1.15). For severe pre-eclampsia, the pooled RR was 0.60 (95\%CI: 0.07-4.73; $\mathrm{I}^{2}=0 \%$ ) (Additional file 2: Appendix 1.16). Eleven studies have reported on the perinatal mortality in both the arms. The pooled RR for early mild pre-eclampsia patients was 1.20 (95\%CI: 0.70 to $2.07 ; \mathrm{I}^{2}=0 \%$ ) (Additional file 2: Appendix 1.17). Four studies have reported perinatal deaths on late onset mild patients, out of which only study reported perinatal deaths with RRof3.12 (95\%CI: 0.13 to $74.80 ; \mathrm{I}^{2}=\mathrm{NA}$ ) (Additional file 2: Appendix 1.18). Five studies reported among early onset severe patients with pooled RR of 3.12 (95\%CI: 0.13-74.80, $\mathrm{I}^{2}=0 \%$ ) (Additional file 2: Appendix 1.19). Eleven studies have reported on the neonatal mortality in both the arms. Out of these, six studies were conducted among mild pre-eclampsia patients ( 2 studies among early onset and 4 among late onset patients). The pooled RR for early onset mild preeclampsia was 1.24 (95\%CI: 0.68 to $\left.2.25 ; \mathrm{I}^{2}=0 \%\right)$ (Additional file 2: Appendix 1.20) and for late onset patients was 3.12 (95\% CI: 0.13-74.80, $\mathrm{I}^{2}=0 \%$ ) (Additional file 2: Appendix 1.21). Five studies were conducted among early severe pre-eclampsia patients. The pooled RR was 1.60 (95\%CI: 0.66 to $3.88 ; \mathrm{I}^{2}=0 \%$ ) (Additional file 2: Appendix 1.22).

Six studies have reported on the risk of respiratory distress syndrome among neonates in both the arms. Out of these, three studies were conducted among late onset mild pre-eclampsia patients. The pooled RR was 2.15 (95\%CI: 1.14 to $4.06 ; \mathrm{I}^{2}=0 \%$ ) (Additional file 2: Appendix 1.23). Three other studies were conducted among early onset severe pre-eclampsia patients. The pooled RR was 1.69 (95\%CI: $1.00-2.85 ; \mathrm{I}^{2}=55 \%$ ) (Additional file 2: Appendix 1.24). Two studies have reported on the risk of seizures among neonates in both the arms. Both the studies were conducted among mild pre-eclampsia patients. However, one study was conducted among early onset patients with RR of 2.57 (95\%CI: $0.27-24.43)$ and one among late onset patients with RR of 3.97 (95\%CI: 0.45-35.30) (Additional file 2: Appendix 1.25 \& 1.26). Nine studies have reported on the risk of small for gestational age (SGA) babies among neonates in both the arms. Five studies were conducted among mild pre-eclampsia patients (one in early onset and four in late onset patients). The pooled RR of late onset patients was 1.19 (95\%CI: 0.73 to $\left.1.94 ; \mathrm{I}^{2}=45 \%\right)$ (Additional file 2: Appendix 1.27) and RR of early onset patients was 1.50 (95\%CI: 0.46-4.89, $\left.\mathrm{I}^{2}=\mathrm{NA}\right)$ (Additional file 2: Appendix 1.28). Four studies were 


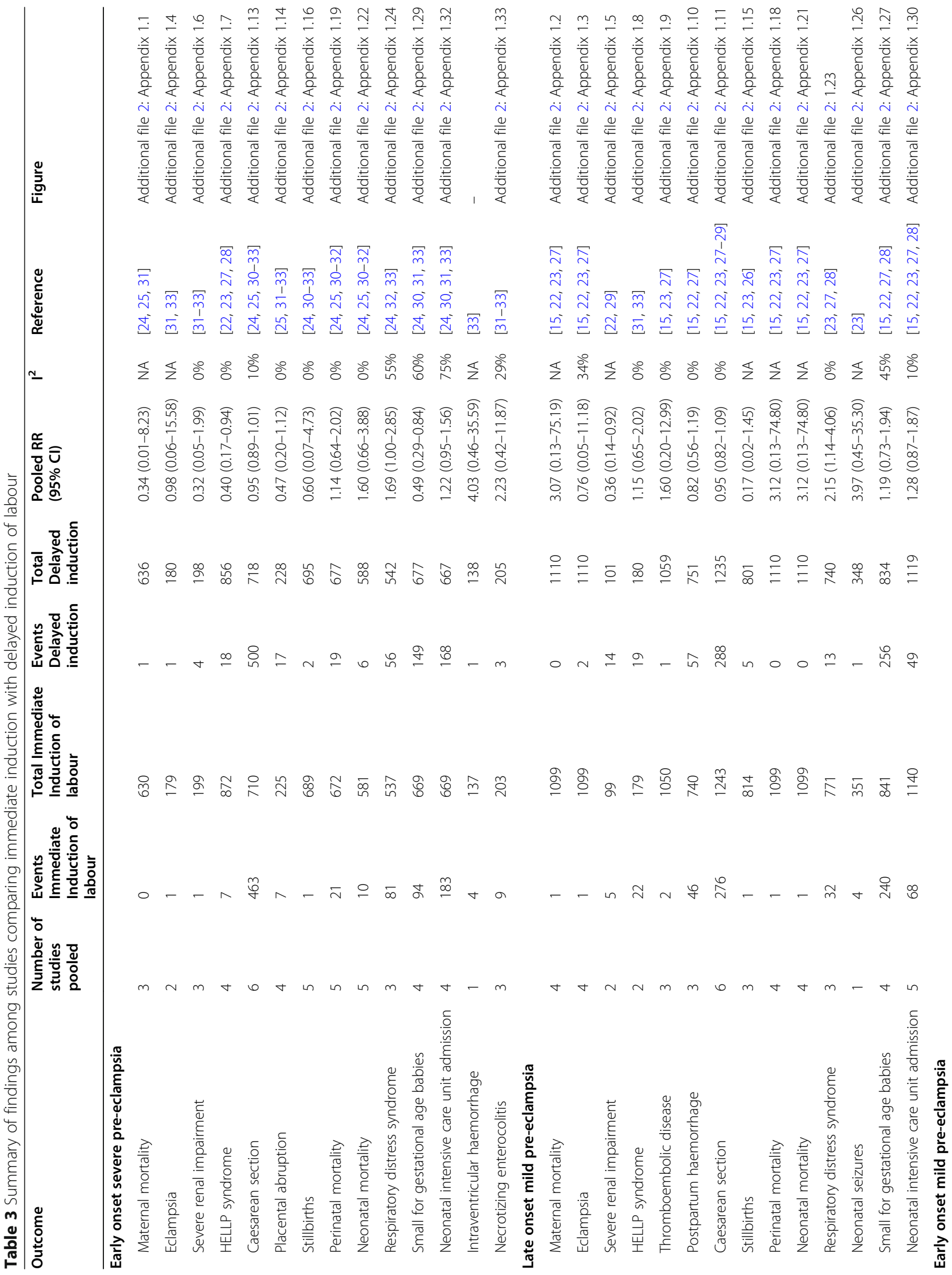




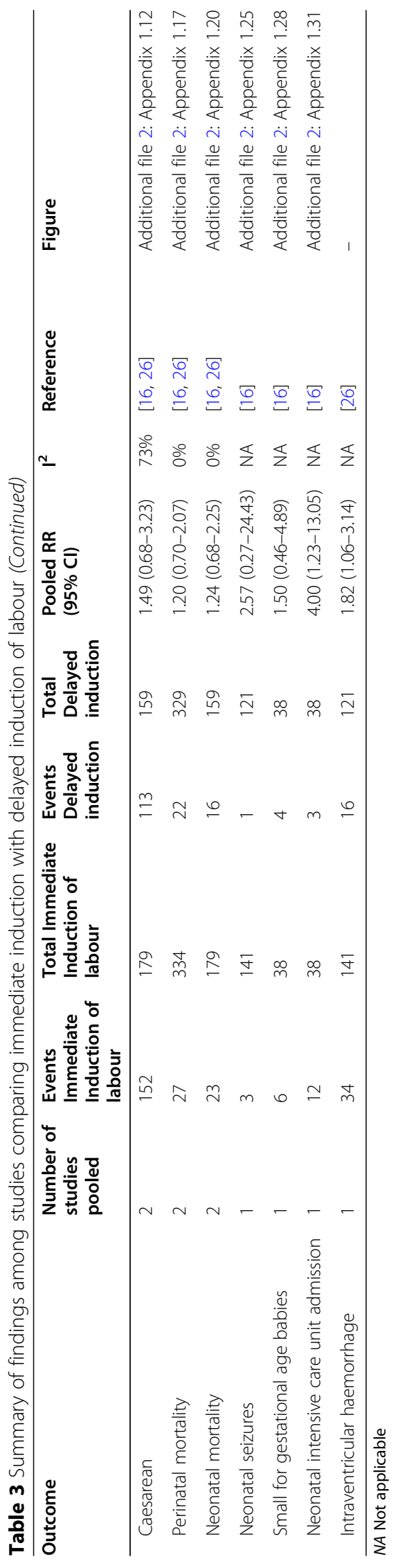


conducted among early onset severe pre-eclampsia patients. The pooled RR was 0.49 (95\%CI: 0.29-0.84; $\mathrm{I}^{2}=60 \%$ ) (Additional file 2: Appendix 1.29).

Ten studies have reported on the neonatal intensive care unit (NICU) admission in both the arms. Six studies were conducted among mild pre-eclampsia patients (one early onset and five late onset preeclampsia patients). The pooled RR for late onset patients was 1.28 (95\% CI: 0.87 to $1.87 ; \mathrm{I}^{2}=10 \%$ ) (Additional file 2: Appendix 1.30) and RR for early onset patients was 4.00 (95\%CI: $1.23-13.05, \mathrm{I}^{2}=\mathrm{NA}$ ) (Additional file 2: Appendix 1.31). Four studies were conducted among early onset severe pre-eclampsia patients. The pooled RR was 1.22 (95\%CI: 0.95-1.56; $\mathrm{I}^{2}=75 \%$ ) (Additional file 2: Appendix 1.32). Two studies have reported on intraventricular haemorrhage in both the arms (one study among early onset severe pre-eclampsia patients and other in late onset mild pre-eclampsia patients). The RR reported in early onset severe patients were 4.03 (95\%CI: 0.46-35.59; $\mathrm{I}^{2}=$ Not applicable) and early onset mild patients were $1.82\left(1.06-3.14 ; \mathrm{I}^{2}=\right.$ Not applicable). Four studies have reported on necrotizing enterocolitis, out of which three were conducted among early onset severe patients and only one among early onset mild patients. The pooled RR for early onset severe patients were 2.23 (95\%CI: 0.42-11.87; $\mathrm{I}^{2}=29 \%$ ) (Additional file 2: Appendix 1.33).

\section{Discussion}

Mothers with hypertensive disorder of pregnancy can be managed with either immediate induction of labour or delayed induction with expectant monitoring of both mother and baby. There are risks and benefits associated with both the type of interventions. Hence, it is important to analyse literature so as to which intervention is associated with better maternal and foetal outcomes.

In all, we identified 14 studies with 4244 participants for our analysis. Majority of the studies were conducted in countries of European region and American region. Most of the included studies had low risk of bias with respect to all the domains except blinding process domains. We did not find any substantial heterogeneity for most of the outcomes in the studies except studies reporting caesarean section rate in both the arms.

Only three outcomes (one maternal and two neonatal outcomes) showed a statistically significant difference between the two interventions. Immediate induction of labour was beneficial to mothers as it had reduced risk of severe renal impairment among late onset mild pre-eclampsia patients. It was also beneficial in neonatal outcome in terms of SGA babies among severe pre-eclampsia patients. Our analysis also demonstrated that delayed induction significantly reduces the risk of neonatal respiratory distress syndrome among late onset mild preeclampsia patients. Our results are similar to the previous Cochrane review conducted by Churchill D et al. in 2018 [34]. In their study too, immediate induction of labour was associated with reduced risk of SGA babies and the risk of neonatal respiratory distress syndrome was found to be lower with delayed induction and expectant management. In our results, no statistically significant difference was noted in any of the other outcomes as the confidence interval crossed the null value in all our remaining analyses. This indicates that evidence on the superiority of one intervention over the other for managing women with hypertensive disorder of pregnancy is limited.

The ACOG guidelines classify mild pre-eclampsia as systolic blood pressure of $140-149 \mathrm{mmHg}$ and/or diastolic pressure of $90-99 \mathrm{mmHg}$, in women with a previously normal blood pressure with proteinuria of $\geq 300 \mathrm{mg} / 24$-h urine collection. On the other hand, severe pre-eclampsia is classified as systolic blood pressure of $>160 \mathrm{mmHg}$ and/or diastolic pressure of $>110 \mathrm{mmHg}$ with severe proteinuria $(2-5 \mathrm{~g} / 24$-h urine collection) [19]. It is important to note that majority of the include studies in our analysis were conducted on early onset severe pre-eclampsia and late onset mild pre-eclampsia. The number of studies pooled for a meta-analysis on maternal and neonatal outcomes for early onset mild pre-eclampsia were limited to just two. While our analysis did not find any difference between the two interventions for incidence of caesarean section, perinatal mortality and neonatal mortality with pooled analysis of the two studies, lack of data significantly limits the ability of our review to derive strong conclusions for this sub-group. In view of this, clinicians should carefully weigh the risk vs benefits of each intervention on a case-to-case basis to achieve optimal maternal and fetal outcomes for patients with early mild pre-eclampsia.

Three other similar reviews have been conducted on this topic, a meta-analysis of RCTs by Cluver $\mathrm{C}$ et al. (2017), Wang Y et al. (2017) and individual participant data meta-analysis by Bernandes et al. (2019) have also reported almost similar findings compared to our review [35-37]. However, the number of studies included in these previous reviews have been limited to only 4-5 trials. In comparison, the current review has analysed 14 studies and comprehensively reviewed the maternal and neonatal morbidity \& mortality outcomes with immediate induction and delayed induction of labour. We believe, the updated evidence shall help healthcare personnel in appropriately choosing the timing of 
induction of labour for the patients with hypertensive disorder of pregnancy.

The major strengths of our study include the comprehensive search of literature and the broad search strategy resulting in inclusion of all studies published on the topic to date. We only included RCTs into our review which enables us to infer causal associations between the intervention and outcomes. Several maternal and neonatal outcomes were analysed in our study thereby providing a comprehensive comparison of the two intervention modalities. The level of inter-study heterogeneity among majority of the maternal or neonatal outcomes was low. Outcomes were stratified based on the disease severity and gestational age of onset to take into account the influence of such confounding factors on the overall outcome.

We are also aware of the limitations of our review. We could not assess the possibility of publication bias for majority outcomes due to the limited number of included studies. Finally, most of the studies included in our review were conducted in European region, which may limit the generalizability of our findings to other geographical areas.

\section{Conclusion}

To summarize, delayed induction of labour with expectant monitoring may not be inferior to immediate induction of labour in terms of maternal and neonatal outcomes. Expectant approach of management for late onset mild pre-eclampsia patients may be associated with decreased risk of neonatal respiratory distress syndrome. Immediate induction of labour among severe pre-eclampsia patients is associated with reduced risk of SGA babies and among mild pre-eclampsia patients, it may be associated with reduced risk of severe renal impairment. Evidence on early onset mild pre-eclampsia was limited to draw strong conclusions.

\section{Supplementary Information}

The online version contains supplementary material available at https://doi. org/10.1186/s12884-020-03407-8.

Additional file 1: Figure S1. Funnel plot checking for publication bias $(n=14)$.

Additional file 2: Appendix 1.1. Forest plot showing the difference in maternal mortality between immediate and delayed induction of labour among early onset severe pre-eclampsia patients. Appendix 1.2. Forest plot showing the difference in maternal mortality between immediate and delayed induction of labour among late onset mild pre-eclampsia patients. Appendix 1.3. Forest plot showing the difference in eclampsia between immediate and delayed induction of labour among late onset mild pre-eclampsia patients. Appendix 1.4. Forest plot showing the difference in eclampsia between immediate and delayed induction of labour among early onset severe pre-eclampsia patients. Appendix 1.5. Forest plot showing the difference in renal failure between immediate and delayed induction of labour among late onset mild pre-eclampsia patients. Appendix 1.6. Forest plot showing the difference in renal failure between immediate and delayed induction of labour among early onset severe pre-eclampsia patients. Appendix 1.7. Forest plot showing the difference in HELLP syndrome between immediate and delayed induction of labour among early onset severe pre-eclampsia patients. Appendix 1.8. Forest plot showing the difference in HELLP syndrome between immediate and delayed induction of labour among late onset mild pre-eclampsia patients. Appendix 1.9. Forest plot showing the difference in thromboembolic disease between immediate and delayed induction of labour among late onset mild pre-eclampsia patients.

Appendix 1.10. Forest plot showing the difference in postpartum haem orrhage between immediate and delayed induction of labour among late onset mild pre-eclampsia patients. Appendix 1.11. Forest plot showing the difference in caesarean section between immediate and delayed induction of labour among late onset mild pre-eclampsia patients. Appendix 1.12. Forest plot showing the difference in caesarean section between immediate and delayed induction of labour among early onset mild pre-eclampsia patients. Appendix 1.13. Forest plot showing the difference in caesarean section between immediate and delayed induction of labour among early onset severe pre-eclampsia patients. Appendix 1.14. Forest plot showing the difference in placental abruption between immediate and delayed induction of labour among early onset severe pre-eclampsia patients. Appendix 1.15. Forest plot showing the difference in stillbirth between immediate and delayed induction of labour among late onset mild pre-eclampsia patients. Appendix 1.16. Forest plot showing the difference in stillbirth between immediate and delayed induction of labour among early onset severe pre-eclampsia patients. Appendix 1.17. Forest plot showing the difference in perinatal mortality between immediate and delayed induction of labour among early onset mild pre-eclampsia patients. Appendix 1.18. Forest plot showing the difference in perinatal mortality between immediate and delayed induction of labour among late onset mild pre-eclampsia patients. Appendix 1.19. Forest plot showing the difference in perinatal mortality between immediate and delayed induction of labour among early onset severe pre-eclampsia patients. Appendix 1.20. Forest plot showing the difference in neonatal mortality between immediate and delayed induction of labour among early onset mild pre-eclampsia patients. Appendix 1.21. Forest plot showing the difference in neonatal mortality between immediate and delayed induction of labour among late onset mild preeclampsia patients. Appendix 1.22. Forest plot showing the difference in neonatal mortality between immediate and delayed induction of labour among early onset severe pre-eclampsia patients. Appendix 1.23. Forest plot showing the difference in neonatal respiratory distress syndrome between immediate and delayed induction of labour among late onset mild pre-eclampsia patients. Appendix 1.24. Forest plot showing the difference in neonatal respiratory distress syndrome between immediate and delayed induction of labour among early onset severe pre-eclampsia patients. Appendix 1.25. Forest plot showing the difference in neonatal seizures between immediate and delayed induction of labour among early onset mild pre-eclampsia patients. Appendix 1.26. Forest plot showing the difference in neonatal seizures between immediate and delayed induction of labour among early onset severe pre-eclampsia patients. Appendix 1.27. Forest plot showing the difference in small for gestational age babies between immediate and delayed induction of labour among late onset mild pre-eclampsia patients. Appendix 1.28. Forest plot showing the difference in small for gestational age babies between immediate and delayed induction of labour among early onset mild pre-eclampsia patients. Appendix 1.29. Forest plot showing the difference in small for gestational age babies between immediate and delayed induction of labour among early onset severe preeclampsia patients. Appendix 1.30. Forest plot showing the difference in neonatal intensive care unit admission rate between immediate and delayed induction of labour among late onset mild pre-eclampsia patients. Appendix 1.31. Forest plot showing the difference in neonatal intensive care unit admission rate between immediate and delayed induction of labour among early onset mild pre-eclampsia patients. Appendix 1.32. Forest plot showing the difference in neonatal intensive care unit admission rate between immediate and delayed induction of labour among early onset severe pre-eclampsia patients. Appendix 1.33. Forest plot showing the difference in necrotizing enterocolitis 
between immediate and delayed induction of labour among early onset severe pre-eclampsia patients

\section{Abbreviations}

CENTRAL: Cochrane Controlled Register of Trials; RR: Risk ratios;

Cls: Confidence intervals; HELLP: Haemolysis, elevated liver enzymes and low platelet; RDS: Respiratory distress syndrome; ICTRP: International Clinical Trials Registry Platform; MeSH: Medical subject heading; PRISMA: Preferred Reporting Items for Systematic Review and Meta-Analysis; RCT: Randomized Controlled Trials; NICU: Neonatal intensive care unit

\section{Acknowledgments}

Not applicable.

\section{Authors' contributions}

$J \mathrm{~L}$ and $\mathrm{SS}$ designed the paper. $J \mathrm{~L}, \mathrm{XS}, \mathrm{QL}, \mathrm{YL}$ and $\mathrm{XQ}$ were involved in literature search and data interpreted. XS, QL and YL were responsible for the data analysis. JL prepared the manuscript. SS edited the manuscript. The authors have read and approved the final manuscript.

\section{Funding}

Not applicable.

\section{Availability of data and materials}

The datasets used and/or analyzed during the current study are available from the corresponding author on reasonable request.

\section{Ethics approval and consent to participate}

Not applicable.

\section{Consent for publication}

Not applicable.

\section{Competing interests}

The authors declare that they have no competing interests.

\section{Author details}

'Department of Obstetrics, The Third Central Hospital of Tianjin, 83 Jintang Road, Hedong District, Tianjin 300170, China. ${ }^{2}$ Tianjin Key Laboratory of Extracorporeal Life Support for Critical Diseases, Tianjin, China. ${ }^{3}$ Artificial Cell Engineering Technology Research Center, Tianjin, China. ${ }^{4}$ Tianjin Institute of Hepatobiliary Disease, Tianjin, China.

\section{Received: 24 April 2020 Accepted: 10 November 2020}

\section{Published online: 26 November 2020}

\section{References}

1. Steegers EAP, von Dadelszen P, Duvekot JJ, Pijnenborg R. Pre-eclampsia. Lancet. 2010;376:631-44

2. Mol BWJ, Roberts CT, Thangaratinam S, Magee LA, de Groot CJM, Hofmeyr GJ. Pre-eclampsia. Lancet. 2016;387:999-1011.

3. Abalos E, Cuesta C, Grosso AL, Chou D, Say L. Global and regional estimates of preeclampsia and eclampsia: a systematic review. Eur J Obstet Gynecol Reprod Biol. 2013;170:1-7.

4. von Dadelszen P, Magee LA. Preventing deaths due to the hypertensive disorders of pregnancy. Best Pract Res Clin Obstet Gynaecol. 2016;36: 83-102.

5. Wallis AB, Saftlas AF, Hsia J, Atrash HK. Secular trends in the rates of preeclampsia, eclampsia, and gestational hypertension, United States, 19872004. Am J Hypertens. 2008;21:521-6.

6. Dahlstrøm BL, Engh ME, Bukholm G, Oian P. Changes in the prevalence of pre-eclampsia in Akershus County and the rest of Norway during the past 35 years. Acta Obstet Gynecol Scand. 2006;85:916-21.

7. Duley L. The global impact of pre-eclampsia and eclampsia. Semin Perinatol. 2009;33:130-7.

8. Khan KS, Wojdyla D, Say L, Gülmezoglu AM, Van Look PF. WHO analysis of causes of maternal death: a systematic review. Lancet. 2006;367:1066-74.

9. Engle W. Tomashek K. Wallman C. "Late-preterm" infants: a population at risk. Pediatrics. 2008;120:1390-401.
10. Leone A, Ersfeld P, Adams M, Schiffer PM, Bucher HU, Arlettaz R. Neonatal morbidity in singleton late preterm infants compared with full-term infants. Acta Paediatr. 2012;101:e6-10.

11. Wang ML, Dorer DJ, Fleming MP, Catlin EA. Clinical outcomes of near-term infants. Pediatrics. 2004;114:372-6.

12. Katz J, Lee AC, Kozuki N, Lawn JE, Cousens S, Blencowe $\mathrm{H}$, et al. Mortality risk in preterm and small-for-gestational-age infants in lowincome and middle-income countries: a pooled country analysis. Lancet. 2013;382:417-25.

13. Seikku L, Gissler M, Andersson S, Rahkonen P, Stefanovic V, Tikkanen M, et al. Asphyxia, neurologic morbidity, and perinatal mortality in immediateterm and Postterm birth. Pediatrics. 2016;137:e20153334. https://doi.org/10. 1542/peds.2015-3334.

14. Yeast JD, Jones A, Poskin M. Induction of labor and the relationship to cesarean delivery: a review of 7001 consecutive inductions. Am J Obstet Gynecol. 1999;180(3 Pt 1):628-33.

15. Boers KE, Vijgen SMC, Bijlenga D, van der Post JAM, Bekedam DJ, Kwee A, et al. Induction versus expectant monitoring for intrauterine growth restriction at term: randomised equivalence trial (DIGITAT). BMJ. 2010;341. https://doi.org/10.1136/bmj.c7087.

16. Hamed HO, Alsheeha MA, Abu-Elhasan AM, Abd Elmoniem AE, Kamal MM Pregnancy outcomes of expectant management of stable mild to moderate chronic hypertension as compared with planned delivery. Int J Gynaecol Obstet. 2014;127:15-20.

17. Royal College of Obstetricians and Gynaecologists. The management of severe pre-eclampsia/eclampsia; 2006. http://isshp.org/wp-content/ uploads/2014/05/rcog.pdf. Accessed 26 Jan 2017.

18. National Institute for Health and Clinical Excellence. Hypertension in pregnancy: the management of hypertensive disorders during pregnancy; 2010. http://guidance.nice.org.uk/cg107. Accessed 26 Jan 2017.

19. American College of Obstetricians and Gynecologists. (ACOG) American College of Obstetricians and Gynecologists' task force on hypertension in pregnancy: hypertension in pregnancy. Obstet Gynecol. 2013;122:1122-31. https://doi.org/10.1097/01.AOG.0000437382.03963.88.

20. Moher D, Liberati A, Tetzlaff J, Altman DG, PRISMA Group. Preferred reporting items for systematic reviews and meta-analyses: the PRISMA statement. PLoS Med. 2009;6:e1000097.

21. Higgins JPT, Green S, Cochrane collaboration, editors. Cochrane handbook for systematic reviews of interventions. Chichester and Hoboken: WileyBlackwell; 2008

22. Bhageerathy $P$, Thomas $V$, Regi A, Jose R. Induction of labour versus conservative management for mild gestational hypertension at term. Int J Reprod Contracept Obstet Gynecol. 2016;5:689-95.

23. Broekhuijsen K, van Baaren G-J, van Pampus MG, Ganzevoort W, Sikkema JM, Woiski MD, et al. Immediate delivery versus expectant monitoring for hypertensive disorders of pregnancy between 34 and 37 weeks of gestation (HYPITAT-II): an open-label, randomised controlled trial. Lancet. 2015:385: 2492-501.

24. Chappell LC, Brocklehurst P, Green ME, Hunter R, Hardy P, Juszczak E, et al. Planned immediate delivery or expectant management for late preterm pre-eclampsia (PHOENIX): a randomised controlled trial. Lancet. 2019;394: 1181-90.

25. Duvekot J, Bax C, Bloemenkamp K, Dijk P, Drongelen JV, Franssen M, et al. 486: temporizing management versus termination of pregnancy in women with severe preeclampsia at 28-34 weeks (TOTEM-trial). Am J Obstet Gynecol. 2015;212:S246.

26. GRIT Study Group. A randomised trial of timed delivery for the compromised preterm fetus: short term outcomes and Bayesian interpretation. Br J Obstet Gynecol. 2003;110:27-32.

27. Koopmans CM, Bijlenga D, Groen H, Vijgen SM, Aarnoudse JG, Bekedam DJ, et al. Induction of labour versus expectant monitoring for gestational hypertension or mild pre-eclampsia after 36 weeks' gestation (HYPITAT): a multicentre, open-label randomised controlled trial. Lancet. 2009;374:979-88.

28. Owens MY, Thigpen B, Parrish MR, Keiser SD, Sawardecker S, Wallace K, et al. Management of preeclampsia when diagnosed between 34-37 weeks gestation: deliver now or deliberate until 37 weeks? J Miss State Med Assoc. 2014;55:208-11.

29. Majeed A, Kundu S, Singh P. Study on induction of labour versus expectant management in gestational hypertension or mild preeclampsia after 36 weeks of gestation. Br J Obstet Gynecol. 2014:121(Suppl 2):118. 
30. Mesbah EMM. Severe preterm preeclampsia: aggressive or expectant management? Med J Cairo Univ. 2003;71:175-82

31. Vigil-De Gracia P, Reyes Tejada O, Calle Miñaca A, Tellez G, Chon VY, Herrarte E, et al. Expectant management of severe preeclampsia remote from term: the MEXPRE Latin study, a randomized, multicenter clinical trial. Am J Obstet Gynecol. 2013;209:425.e1e8. https://doi.org/10.1016/j.ajog.2013.08.016 [PubMed] [CrossRef] [Google Scholar].

32. Odendaal HJ, Pattinson RC, Bam R, Grove D, Kotze TJWW. Aggressive or expectant Management for Patients with Severe Preeclampsia between 2834 weeks' gestation: a randomized controlled trial. Obstet Gynecol. 1990;76: 1070-5 [PubMed] [Google Scholar].

33. Sibai BM, Mercer BM, Schiff E, Friedman SA. Aggressive versus expectant management of severe preeclampsia at 28 to 32 weeks' gestation: as randomized controlled trial. Am J Obstet Gynecol. 1994;171:818-22.

34. Churchill D, Duley L, Thornton JG, Moussa M, Ali HSM, Walker KF, et al. Interventionist versus expectant care for severe pre-eclampsia between 24 and 34 weeks' gestation. Cochrane Database Syst Rev. 2018;2018(10): CD003106.

35. Cluver C, Novikova N, Koopmans CM, West HM. Planned immediate delivery versus expectant management for hypertensive disorders from 34 weeks gestation to term. Cochrane Database Syst Rev. 2017;1:CD009273.

36. Bernardes TP, Zwertbroek EF, Broekhuijsen K, Koopmans C, Boers K, Owens $M$, et al. Delivery or expectant management for prevention of adverse maternal and neonatal outcomes in hypertensive disorders of pregnancy: an individual participant data meta-analysis. Ultrasound Obstet Gynecol. 2019:53:443-53.

37. Wang Y, Hao M, Sampson S, Xia J. Elective delivery versus expectant management for pre-eclampsia: a meta-analysis of RCTs. Arch Gynecol Obstet. 2017;295(3):607-22.

\section{Publisher's Note}

Springer Nature remains neutral with regard to jurisdictional claims in published maps and institutional affiliations.

Ready to submit your research? Choose BMC and benefit from:

- fast, convenient online submission

- thorough peer review by experienced researchers in your field

- rapid publication on acceptance

- support for research data, including large and complex data types

- gold Open Access which fosters wider collaboration and increased citations

- maximum visibility for your research: over $100 \mathrm{M}$ website views per year

At $\mathrm{BMC}$, research is always in progress.

Learn more biomedcentral.com/submissions 